\title{
Surface Micromachined Linear Electrostatic Stepper Motor
}

\author{
N.R. Tas ${ }^{l}$, A.H. Sonnenberg ${ }^{l}$, A.F.M. Sander ${ }^{2}$ and M.C. Elwenspoek ${ }^{1}$ \\ ${ }^{1} M E S A$ Research Institute, University of Twente \\ P.O. Box 217, 7500 AE Enschede, the Netherlands \\ ${ }^{2}$ Philips Research, WA 1-2 10, Prof. Holstlaan 4, \\ 5656 AA Eindhoven, the Netherlands
}

\begin{abstract}
A linear electrostatic stepper motor has been designed and fabricated in a single mask surface micromachining process.. It consists of two drive units that alternately generate a step to move the shuttle. The friction and adhesion in the clamps has been measured. A friction coefficient of $0.8 \pm 0.3$ have been found. The adhesion in the clamp is just low enough to release the clamping shoe. Stepping cycles have been performed succesfully to obtain a deflection of $15 \mu \mathrm{m}$, with steps in the order of $2 \mu \mathrm{m}$. The deflection is limited by the suspension. The generated force equals $3 \mu \mathrm{N}$ at $40 \mathrm{~V}$ driving voltage.
\end{abstract}

\section{INTRODUCTION}

Linear motors that apply a walking motion are able to add small steps in order to obtain large translations [14]. The possibility to combine a high resolution with a large total stroke makes them attractive for applications like high density data storage or scanning microscopy. The electrostatic stepper motor presented here is fabricated in a very simple single mask surface micromachining process. The motor is made in a single level of $5 \mu \mathrm{m}$ polysilicon and will be operated laterally. Moving structures are made with the sacrificial layer technique. The motor consists of two drive units that alternately generate a step to move a shuttle. The main goal of the work presented here is to investigate the stepwise motion on microscale. The experiments therefore focus on the friction and adhesion in the clamps.
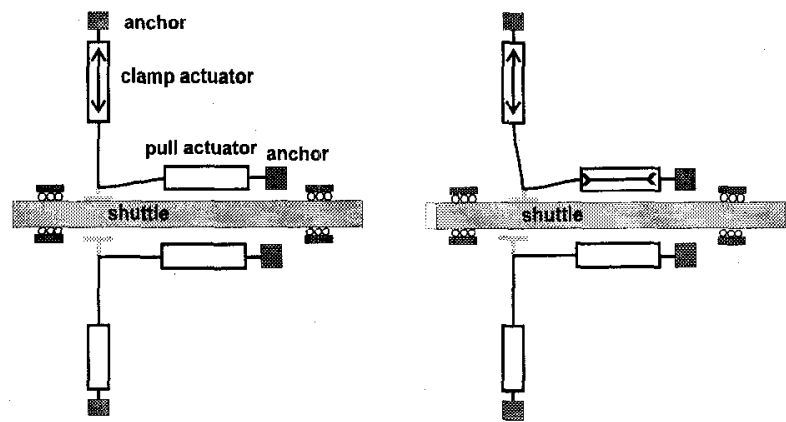

\section{DESIGN}

\subsection{Principle of operation}

Figure 1 shows the principle of operation of the stepper motor. Drive unit 1 first clamps its shoe against the shuttle and pulls one step. Next, drive unit 2 takes over the clamping and pulls a next step.

\subsection{Single mask design}

The clamp force can be generated by applying a voltage difference between the clamp shoe and the shuttle [4]. A problem with this approach can be that there is a voltage difference between either the shuttle and the substrate or the clamp shoe and the substrate. To avoid electrostatic pull-down of one of these moving parts to the substrate, electrical shields are needed, unless the charged structures are made very stiff [5]. We have investigated the application of separate clamp actuators, in which all moving parts are kept at the same potential as the substrate in order to avoid electrostatic pull-down of released structures. Drive voltages are applied to the stator poles of the electrostatic actuators. Therefore electrical shields underneath moving parts are not required, which saves one mask step. By using a timed sacrificial layer etch, the distinction between anchored and released parts can be made. This saves an additional mask step. All released parts are made narrower than 10 $\mu \mathrm{m}$, and all anchored parts wider than $10 \mu \mathrm{m}$.

Figure 1: Principle of operation. Drive unit l first clamps its shoe against the shuttle and pulls one step. Next, Drive unit 2 takes over the clamping and pulls a next step. 


\subsection{Actuators}

The clamp and pull actuators are formed by arrays of gap-closing parallel plate capacitors (figure 2). The gapclosing array actuators combine a high energy density by means of multiple small gaps, with a large change of the actuator volume per unit deflection. This combination explains the relative high force output of this type of actuators.

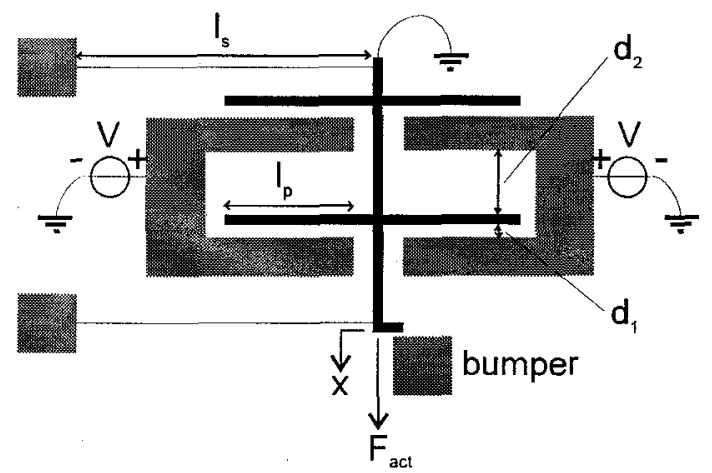

Figure 2: An array of two parallel plate gap-closing actuators. The moving part is drawn black, the anchored part is dark grey. $F_{a c t}$ is the external force that makes equilibrium. The forward initial gap-size is $d_{l}$, the backward initial gap-size is $d_{2}$. The bumper protects the actuator against short-circuit. The drive voltage is applied between the stator plates (grey) and the moveable plates (black). The moveable part is kept on the same potential as the substrate.

The two parallel suspension springs serve as a linear guidance for the actuator array. Residual tensile stress in the polysilicon will be released in this configuration. The actuators are voltage controlled. Therefore, $F_{\text {act }}$ is found from the Legendre transform of the energy function $W$ according to $W^{*}=W-U \cdot Q$ :

$W^{*}(V, x)=-\frac{1}{2} \cdot \frac{\varepsilon \cdot A \cdot V^{2}}{d_{1}-x}+\frac{1}{2} \cdot k \cdot x^{2}$

Where $A$ is the total overlapping area of the actuator plates, $d_{1}$ is the initial gap, $V$ is the applied voltage between the plates, $\varepsilon$ is the permittivity of air, $x$ is the actuator deflection and $k$ is the suspension stiffness. The backward electrostatic force has been neglected in Eq. (1). The external force that makes equilibrium, $F_{\text {act }}$, equals the derivative of the $W^{*}$ with respect to $x$ :

$F_{a c t}=-\frac{1}{2} \cdot \frac{\varepsilon \cdot A \cdot V^{2}}{\left(d_{1}-x\right)^{2}}+k \cdot x$

If the electrostatic attraction force increases faster with $x$ than the restoring spring force, then the actuator becomes unstable and will make a full stroke.The voltage at which the unloaded actuator becomes unstable is found by setting the first and second derivative of $W^{*}$ equal to zero and solving for $V$ :

$V_{p i}=\sqrt{\frac{8 \cdot k \cdot d^{3}}{27 \cdot A \cdot \varepsilon}}$

The suspension stiffness (two parallel springs) equals [6]:

$k=2 \cdot \frac{E \cdot h \cdot w^{3}}{l_{s}^{3}}$

Where $l_{\mathrm{s}}=200 \mu \mathrm{m}$ is the length of the springs, $h=5$ $\mu \mathrm{m}$ is the height of the springs, $w=2 \mu \mathrm{m}$ is the width of the springs and $E=150 \mathrm{GPa}$ is the Young's modulus. This yields a suspension stiffness $k=1.5 \mathrm{~N} / \mathrm{m}$. The actuator area $A$ is given by $n \cdot h \cdot l_{\mathrm{p}}$ where $l_{\mathrm{p}}=100 \mu \mathrm{m}$ is the overlapping plate length and $n$ is the number of plates. For the pull actuator, which creates the step, we have chosen $n=7$ plates, and $d_{1}=3 \mu \mathrm{m}$, which gives $V_{\mathrm{pi}}=20 \mathrm{~V}$. The clamp actuator has got $n=14$ plates, and $d_{1}=4 \mu \mathrm{m}$, which corresponds with $V_{\mathrm{pi}}=21 \mathrm{~V}$. The net actuator force at $40 \mathrm{~V}$ actuation voltage as a function of the actuator deflection is shown in figure 3 . Both the forward and backward electrostatic forces are included in the evaluation. For $n=7$ plates, $d_{1}=3 \mu \mathrm{m}$ and $d_{2}=7$ $\mu \mathrm{m}\left|F_{\text {act }}\right|$ is larger than $2 \mu \mathrm{N}$. For $n=14$ plates, $d_{1}=4$ $\mu \mathrm{m}$ and $d_{2}=6 \mu \mathrm{m},\left|F_{\text {act }}\right|$ is larger than $2 \mu \mathrm{N}$.

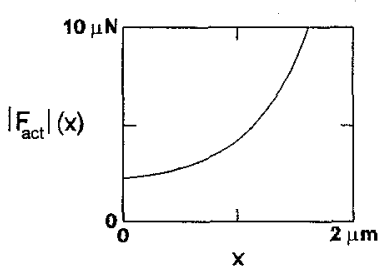

a)

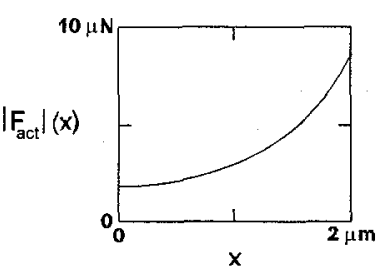

b)
Figure 3: Actuator force as a function of actuator deflection. a) Pull actuator, $n=7, d_{I}=3 \mu \mathrm{m}$ and $d_{2}=7$ $\mu m$. b) Clamp actuator, $n=14, d_{1}=4 \mu m$ and $d_{2}=6$ $\mu m$.

The maximum stroke of each actuator is limited by a bumper that protects it against short-circuit by collision of the plates. The bumper in the pull actuator allows a maximum stroke of $2 \mu \mathrm{m}$. The clamp actuator has a maximum stroke of $3 \mu \mathrm{m}$.

\subsection{Drive units}

A drive unit consists of a clamp actuator, a pull actuator a clamp shoe, and elastic beams that connect the actuators to the shoe (figure 4). The beam that connects the clamp actuator to the shoe is stiffened in the middle to increase the buckling load. A stiffened beam of 
length $7 / 6 \cdot l$ as drawn in figure 5 has a buckling load $F_{x, \max }$ that is 9 times larger than a simple beam of length $l$ and width $w$, while the stiffness $\mathrm{F}_{\mathrm{y}} / y$ is almost the same: $108 / 109$ times the stiffness of the simple beam [7].

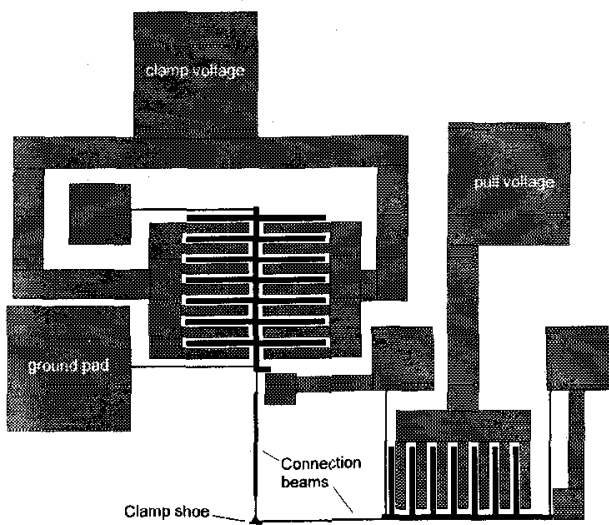

Figure 4: Layout of a drive unit, consisting of a clamp actuator, a pull actuator both connected to a clamp shoe by means of elastic beams. All moveable parts have been drawn black, all fixed parts grey

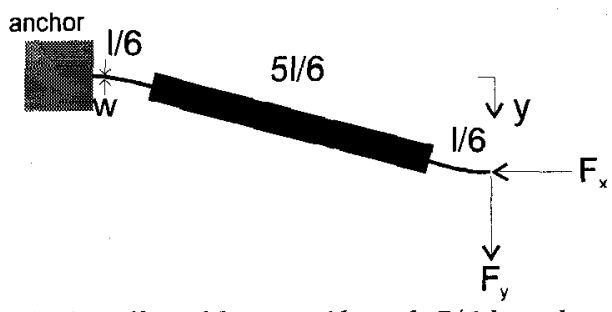

Figure 5: A stiffened beam of length 7/6.l as drawn has a buckling load $F_{x, \max }$ that is 9 times larger than a simple beam of length $l$ and width $w$, while the stiffness $F_{y} y$ is almost the same: 108/109 times the stiffness of the simple beam [7]

\subsection{Complete Motor}

The complete motor consists of two drive units, one on each side of the shuttle (figure 8). The suspension of the shuttle is loaded in the $y$-direction by the drive units (see figure 6) from both sides. Therefore, it has been designed to be both stiff and stable in this direction. Figure 6 shows the layout of the shuttle plus suspension. Holes have been made in the shuttle to minimize the under etch distance for the timed release etch. The suspension springs have been stiffened in the middle to avoid buckling. The total length of the suspension beams is $7 / 6 \times 400 \mu \mathrm{m}$, yielding a stiffness $k_{\mathrm{x}}=0.19$ $\mathrm{N} / \mathrm{m}(E=150 \mathrm{GPa}, w=2 \mu \mathrm{m}, h=5 \mu \mathrm{m})$. A deflection $\Delta x$ in $x$-direction introduces a small displacement $\Delta y \cong$ $\Delta x^{2} / 2 l_{\mathrm{s}}$ in $y$-direction. This leads to a reduction of the clamp force at one side, because the closed-gap size in the clamp actuator becomes larger. For example, a shuttle displacement $\Delta x=18 \mu \mathrm{m}$ gives a displacement $\Delta y=0.4 \mu \mathrm{m}$.

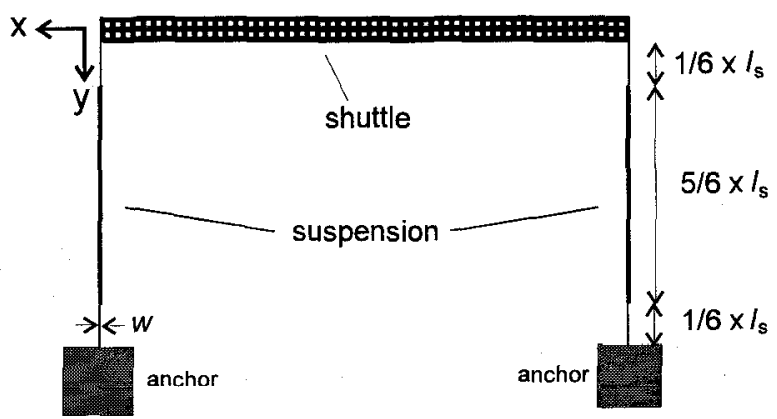

Figure 6: Layout of the shuttle and its suspension springs. The moving part has been drawn black, the anchors dark grey. The suspension springs are stiffened in the middle to increase the buckling load. Holes are etched in the shuttle in order to keep the under etch distance small.

\section{FABRICATION}

The motor is fabricated on a $380 \mu \mathrm{m}$ thick 3 inch silicon wafer, p-type 5-10 $\Omega \mathrm{cm}$. First a $2 \mu \mathrm{m}$ thick thermal oxide is grown, followed by a $5 \mu \mathrm{m}$ thick polysilicon layer grown by LPCVD at a temperature of $590^{\circ} \mathrm{C}$, a pressure of $250 \mathrm{~m}$ Torr and a silane flow of $50 \mathrm{sccm}$. The polysilicon layer is doped with boron by solid source indiffusion for one hour at $1100^{\circ} \mathrm{C}$. Next, a 1.2 $\mu \mathrm{m}$ PECVD oxide mask layer is deposited on top of the polysilicon. To avoid a large stress gradient in the polysilicon it is important to obtain a homogeneous distribution of the boron. Therefore, a post-anneal step is carried out at $1100^{\circ} \mathrm{C}$ for 3 hours under a continuous nitrogen flow. Next, the PECVD oxide mask is patterned by RIE in a $\mathrm{CHF}_{3} / \mathrm{O}_{2}$ gas mixture. The $5 \mu \mathrm{m}$ thick polysilicon is etched anisotropically by RIE in a $\mathrm{SF}_{6} / \mathrm{CHF}_{3} / \mathrm{O}_{2}$ plasma. Finally, the $2 \mu \mathrm{m}$ thick sacrificial oxide is etched for 5 minutes in $50 \% \mathrm{HF}$. After rinsing with IPA and cyclohexane the structure is dried by means of freeze drying of the cyclohexane [8]. By a timed etch of the sacrificial layer it is possible to under etch only narrow structures (figure 7). Figure 8 shows a microscope picture of the complete motor. Figure 9 shows a SEM-picture of a clamp shoe and two elastic drive beams.

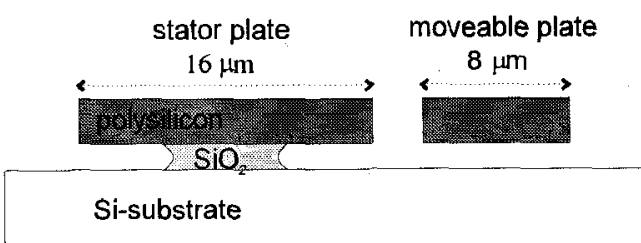

Figure 7: Narrow structures are released selectively, by a timed oxide etch in $\mathrm{HF}$. 


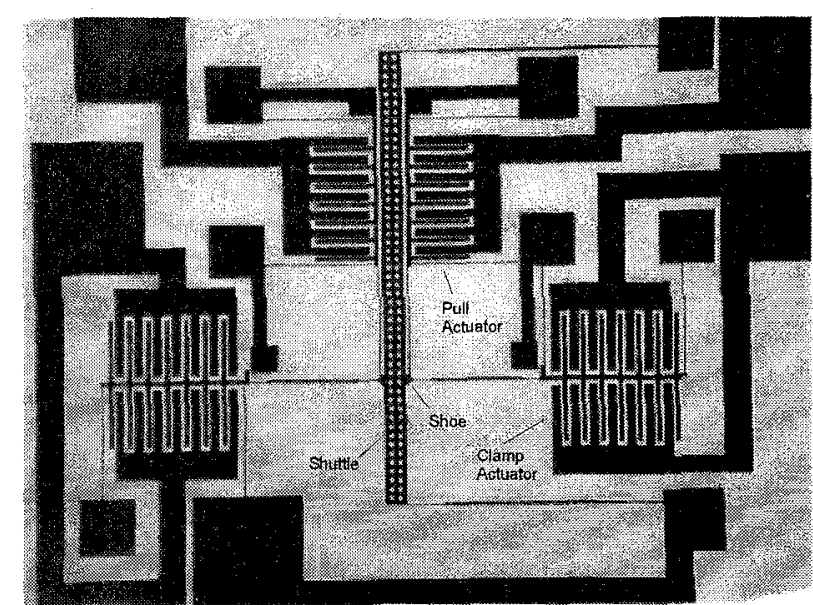

Figure 8: Microscope picture of a fabricated motor $\left(1 \times 1 \mathrm{~mm}^{2}\right)$. The motor consists of a shuttle and two drive units at both sides of the shuttle. Each drive unit consists of a clamp actuator, a pull actuator, a clamp shoe and elastic beams that connect the actuators and the shoe.

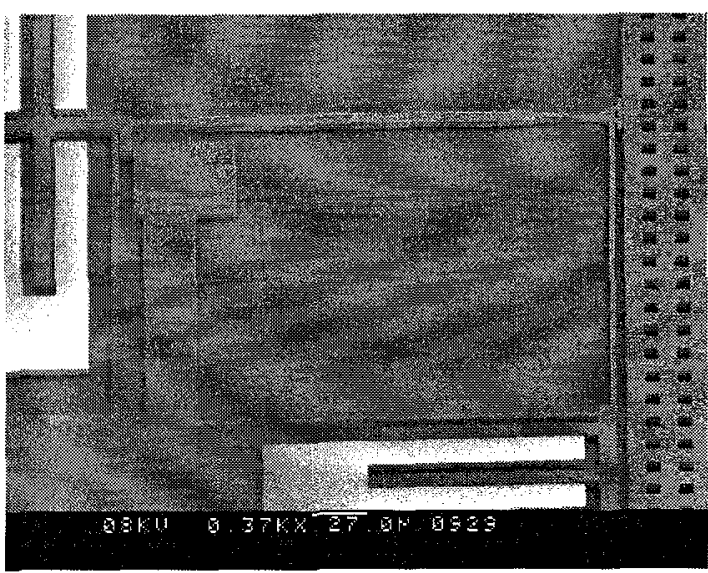

Figure 9: SEM photograph of a clamp shoe and two elastic drive beams. The beam connecting the shoe and the clamp actuator is stiffened in the middle to increase the buckling load. Due to charging of the actuators in the SEM, the shoe is clamped against the shuttle.

\section{TEST OF THE CLAMPS}

For proper operation of the motor, it is important that there is high enough friction if the clamps are activated, and that the clamp shoe releases from the shuttle if the clamps are made inactive. In order to test this, we have fabricated a friction meter on the same wafer as the motors. It consists of a drive unit that clamps its shoe against a rigid wall (figure 10). The measurements have been done using a clamp actuator with 30 plates, and a pull actuator with 15 plates. Friction is measured by pushing the shoe against the wall with a defined force, and measuring the pull force at the onset of slip.

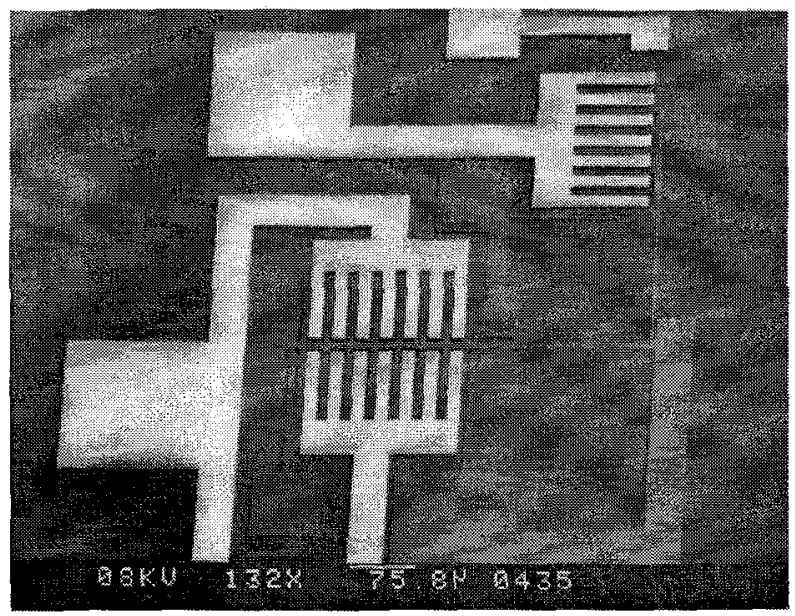

Figure 10: A friction meter. It consists of a drive unit that clamps its shoe against a rigid wall. Friction is determined by measuring the pull force at the onset of slip of the shoe along the wall. The stator poles of the pull and clamp actuator are white due to charging in the SEM.

The total clamp force consists of the externally applied clamp force plus the interfacial adhesion force. We assume that the friction force is proportional to the totally applied clamp force:

$$
F_{\text {fric }}=\mu \cdot\left(F_{\text {ext }}+F_{a d h}\right)
$$

From the measurement we can estimate the adhesion of the shoe to the wall by looking at the friction force at zero applied external force. The external clamp force consists of the generated electrostatic clamp force minus the spring force, due to the stiffness of the suspension and the drive beam connected to the pull actuator.

$$
F_{\text {ext }}=F_{\text {elec }}-F_{\text {spring }}
$$

The restoring spring force during clamping equals $7 \pm 2$ $\mu \mathrm{N}$. This has been calculated from the stiffness of the suspension and the pull connection beam, times the initial gap between the shoe and the shuttle. Figure 11 shows the forces working on the clamp shoe.

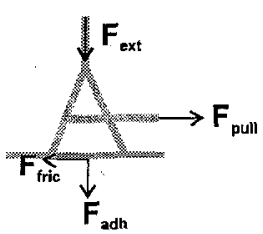

Figure 11: The forces working on a clamp shoe. $F_{\text {ext }}$ is the effective external applied clamp force. $F_{\text {adh }}$ is the adhesion force of the shoe against the wall, which we call the internal clamp force. $F_{\text {pull }}$ is the applied pull force, which in the no slip state makes balance with the friction force $F_{\text {fric- }}$ 
To determine the external clamp force, we need to know the generated electrostatic clamp force $F_{\text {elec }}$. This force can be calculated from the voltage applied on the clamp actuator, $V_{\text {clamp }}$ :

$$
F_{\text {elec }}=\alpha_{\text {clamp }} \cdot V_{\text {clamp }}^{2}
$$

Where $\alpha_{\text {clamp }}$ is the conversion factor from actuator voltage to force $\left[\mathrm{N} / \mathrm{V}^{2}\right]$ which depends on the actuator geometry as follows:

$$
\alpha_{\text {clamp }}=\frac{\varepsilon \cdot A_{c 1}}{2 \cdot\left(d_{c 1}-g\right)^{2}}-\frac{\varepsilon \cdot A_{c 2}}{2 \cdot\left(d_{c 2}+g\right)^{2}}
$$

Where $A_{\mathrm{cl}}=1.5 \times 10^{4}(\mu \mathrm{m})^{2}$ is the forward clamp actuator area, $A_{\mathrm{c} 2}=1.4 \times 10^{4}(\mu \mathrm{m})^{2}$ is the backward clamp actuator area, $d_{\mathrm{c} 1}=3.6 \pm 0.2 \mu \mathrm{m}$ is the forward initial gap of the clamp actuator, $d_{\mathrm{c} 2}=5.2 \pm 0.2 \mu \mathrm{m}$ is the backward initial gap of the clamp actuator and $g=$ $1.4 \pm 0.2 \mu \mathrm{m}$ is the initial gap between the shoe and the shuttle (figure 12). The gaps have been measured in the SEM. Due to the uncertainty in the gap sizes, an uncertainty in the conversion factor exists:

$$
\alpha_{\text {clamp }}=(1.2 \pm 0.3) \times 10^{-8} \mathrm{~N} / \mathrm{V}^{2} \text {. }
$$

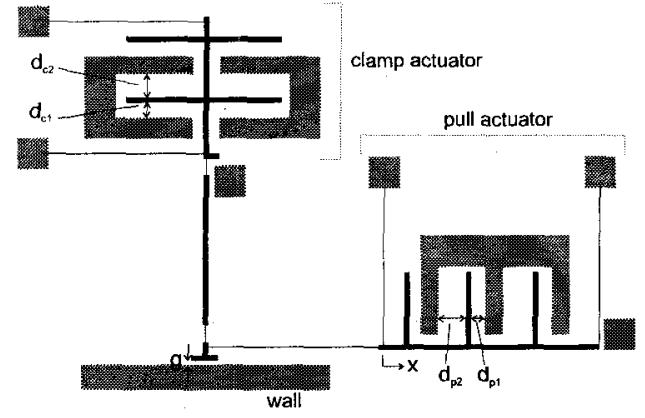

Figure 12: Layout of the friction meter. $d_{c l}$ is the forward initial gap of the clamp actuator, $d_{\mathrm{c} 2}$ is the backward initial gap of the clamp actuator, $d_{p 1}$ is the forward initial gap of the pull actuator, $d_{p 2}$ is the backward initial gap of the pull actuator and $g$ is the initial gap between the shoe and the shuttle.

The friction force is measured by determining the pull voltage at the onset of slip. The shoe is clamped to the shuttle while the pull actuator is in rest. Therefore the spring force of the suspension of the pull actuator is zero, and for the friction force as a function of the applied pull voltage we can write:

$$
F_{\text {fric }}=\alpha_{\text {pull }} \cdot V_{\text {pull }}^{2}
$$

Where $\alpha_{\text {pull }}$ is the conversion factor from actuator voltage to force $\left[\mathrm{N} / \mathrm{V}^{2}\right]$ which depends on the actuator geometry as follows: $\alpha_{\text {pull }}=\frac{\varepsilon \cdot A_{p 1}}{2 \cdot d_{p 1}^{2}}-\frac{\varepsilon \cdot A_{p 2}}{2 \cdot d_{p 2}^{2}}$

Where $A_{\mathrm{pl}}=7.5 \times 10^{3}(\mu \mathrm{m})^{2}$ is the active forward pull actuator area, $A_{\mathrm{p} 2}=7.0 \times 10^{3}(\mu \mathrm{m})^{2}$ is the active backward pull actuator area, $d_{\mathrm{pl}}=2.7 \pm 0.2 \mu \mathrm{m}$ is the forward initial gap of the pull actuator and $d_{\mathrm{p} 2}=6.2 \pm$ $0.2 \mu \mathrm{m}$ is the backward initial gap of the pull actuator. Due to the uncertainty in the gap sizes, an uncertainty in the conversion factor exists: $\alpha_{\text {pull }}=(3.8 \pm 0.8) \times 10^{-9}$ $\mathrm{N} / \mathrm{V}^{2}$. In order to create a visible slip of the shoe along the wall, it is important that the electrostatic forces increase faster than the restoring spring force during slip. In the friction meter used this condition is met for pull-voltages larger than $22 \mathrm{~V}$. Figure 13 shows the measured pull voltages squared at the onset of slip, as a function of the applied clamp voltage squared. The measurements have been carried out at a relative humidity of the air of $35 \pm 5 \%$. The voltages have been converted into forces by using the conversion factors $\alpha_{\text {pull }}$ and $\alpha_{\text {clamp. }}$. The grey area in figure 14 is the region between the line that is formed by taking the smallest $\alpha_{\text {pull }}$ and the largest $\alpha_{\text {clamp }}$ and the line that is formed by taking the largest $\alpha_{\text {pull }}$ and the smallest $\alpha_{\text {clamp. }}$ The measured forces are within this region.

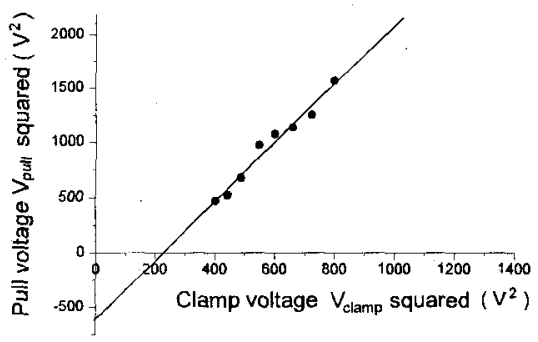

Figure 13: The measured pull voltages squared at the onset of slip, as a function of the applied clamp voltage squared. The measurements have been carried out at a relative humidity of the air of $35 \pm 5 \%$.

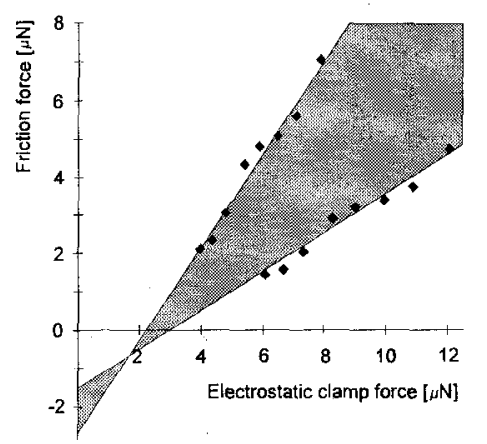

Figure 14: The measured static friction force in the shoe/shuttle interface as a function of the electrostatic clamp force generated in the clamp actuator. The uncertainty in the applied forces is caused by uncertainty in the gaps in the actuators. 
From the slope of the graph a friction coefficient $\mu=$ $0.8 \pm 0.3$ has been determined. This indicates that in the motor a clamp force slightly larger than the pull force is sufficient. The adhesion of the clamp shoe to the shuttle can be estimated from the friction force at zero applied electrostatic clamp force $\left(F_{\text {elec }}=0\right)$. Using eq. (5) and (6) it follows that: $\mathrm{F}_{\text {adh }}=F_{\text {fric }}(0) / \mu+F_{\text {spring }}=5 \pm 4 \mu \mathrm{N}$. This indicates that the adhesion force is only slightly smaller than the restoring spring force. This corresponds with our observations that on moderate humid days (30$40 \% \mathrm{RH}$ ) the adhesion is low enough to release the shoes in the stepping cycle. We also observed that on humid days the shoes sometimes sticked to the shuttle. Apparently, the adhesion depends on the relative humidity of air, which might be explained by an increased capillary condensation effect at higher humidity levels [5].

\section{TEST OF THE MOTOR}

Figure 15 shows the voltage pattern applied to the actuators. The voltages have been applied to the stator poles of the actuators. All moving structures as well as the substrate have been grounded.

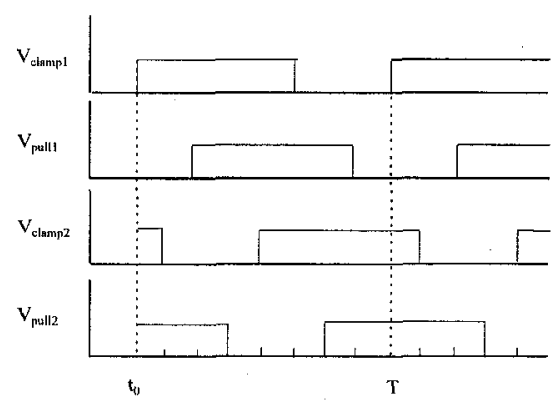

Figure 15: Voltage pattern applied to the actuators.

A complete stepping cycle has been performed successfully at a potential of $40 \mathrm{~V}$ and a cycle frequency of $1 \mathrm{~Hz}$. Steps have been added to obtain a total displacement of $15 \mu \mathrm{m}$, limited by the suspension. Based on the geometry of the suspension of the shuttle, this corresponds with a generated force of $3 \mu \mathrm{N}$. The effective step size decreases from $2 \mu \mathrm{m}$ to zero with increasing shuttle displacement due to slip, mainly in one of the clamps. The reason for this is that the clamp force reduces due to the sideways displacement of the shuttle as a consequence of the used suspension (see section 2.5). Figure 16 shows a picture taken from the video of the motor in operation. Note the deflection of the shuttle suspension.

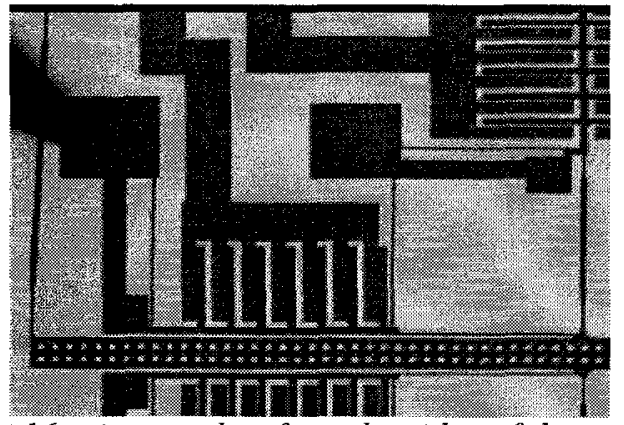

Figure 16: picture taken from the video of the motor in operation. Note the deflection of the shuttle suspension.

\section{CONCLUSIONS}

A linear electrostatic stepper motor has been realized in a single mask surface micromachining process. A friction coefficient of $0.8 \pm 0.3$ has been measured in the clamp of a drive unit. The friction measurements indicate that there is significant adhesion in the clamp. A stepping cycle has been performed successfully, yielding a total stroke of $15 \mu \mathrm{m}$ with a step size of about $2 \mu \mathrm{m}$, decreasing to zero. The generated force is $3 \mu \mathrm{N}$ at $40 \mathrm{~V}$ driving voltage.

\section{ACKNOWLEDGEMENT}

This research is carried out in coorporation with Philips Research. The authors would like to thank M.P. Koster of Philips Center for Manufacturing Technology for helpfull discussions on the mechanical design, D. Blank and $\mathrm{H}$. Visscher for valuable discussions on design and friction measurements. Also they like to thank A. Kooij for making the mask, and B. Otter for making the SEMphotographs.

\section{REFERENCES}

[1] Akiyama T., Shono K.,"Controlled stepwise motion in polysilicon microstructures", J. Microelectromechanical Syst., vol. 2-3, 1993, pp. 106-110.

[2] M.P. Koster,"A Walking Piezo Motor", Proc. Actuator 94 Conf., Bremen, June 1994, pp. 144-148.

[3] N.R. Tas, Legtenberg R., Berenschot J.W., Elwenspoek M.C., Fluitman J.H.J, "The electrostatic shuffle motor", Proc. Micromechanics Europe Workshop 1995, Copenhagen, Denmark, pp. 128-131.

[4] R. Yeh, E.J.J. Kruglick, K.S.J. Pister,"Microelectromechanical components for articulated microrobots", Proc. Transducers '95, Vol. 2, June 1995, pp. 346-349.

[5] N.R.Tas, A.H. Sonnenberg, H.V. Jansen, R. Legtenberg, M.C. Elwenspoek,"Stiction in surface micromachining", Journal of Micromechanics and Microengineering, in press.

[6] Gere J.M. and Timoshenko S.P.,"Mechanics of Materials", 3rd ed., Chapman \& Hall, London, 1991.

[7] Koster M.P.,"Constructieprincipes", ISBN 9036508320, University of Twente Press, Enschede, The Netherlands, 1996.

[8] Legtenberg R.,"Electrostatic actuators fabricated by surface micromachining techniques", Thesis University of Twente, ISBN 90-3650796-0, 1996. 